\title{
THE
}

9-1-1995

\section{Statistical Quasiparticles in Transverse Dynamics of Gases}

\author{
A. E. Meyerovich \\ University of Rhode Island, sfo101@uri.edu \\ S. Stepaniants \\ University of Rhode Island \\ F. Laloë
}

Follow this and additional works at: https://digitalcommons.uri.edu/phys_facpubs

Terms of Use

All rights reserved under copyright.

\section{Citation/Publisher Attribution}

Meyerovich, A. E., Stepaniants, S., \& Laloë, F. (1995). Statistical Quasiparticles in Transverse Dynamics of Gases. Phys. Rev. B., 52(9), 6808-6821. doi: 10.1103/PhysRevB.52.6808

Available at: http://dx.doi.org/10.1103/PhysRevB.52.6808

This Article is brought to you for free and open access by the Physics at DigitalCommons@URI. It has been accepted for inclusion in Physics Faculty Publications by an authorized administrator of DigitalCommons@URI. For more information, please contact digitalcommons-group@uri.edu. 


\title{
Statistical quasiparticles in transverse dynamics of gases
}

\author{
A. E. Meyerovich and S. Stepaniants \\ Department of Physics, University of Rhode Island, Kingston, Rhode Island 02881 \\ F. Laloë \\ Laboratoire Kastler-Brossel, l'Ecole Normale Superieure, 24 Rue Lhomond, Paris 75231, France
}

(Received 16 December 1994; revised manuscript received 24 March 1995)

\begin{abstract}
We analyze the validity of the Fermi-liquid approach to transverse dynamics of spin-polarized gases at arbitrary temperatures. We demonstrate that the diagrammatic kinetic equation for transverse processes can be formulated as a simpler, but completely equivalent equation in terms of "statistical quasiparticles." The equation includes all coherent and dephasing molecular-field terms as well as the dissipative collision integral up to the second order. Beyond the second order, the results become very complicated, and a quasiparticle approach loses its attraction. We give the expressions for the effective interaction function and collision integral for statistical quasiparticles, applicable at all temperatures, and discuss the implications of this concept at high temperatures. The interaction function contains anomalous pole terms which do not exist in equations for longitudinal dynamics. This provides a somewhat unexpected interpretation for zero-temperature dissipative processes, observed recently in spin dynamics, and for controversial molecular-field terms (the so-called $I_{2}$ terms) as imaginary (pole) and real (principal) parts of the quasiparticle interaction function. These molecular-field terms with complicated analytical structure do not vanish completely, as was assumed earlier, in the Boltzmann region, but contribute to higher-order density terms. With an emphasis on quantum gases, we discuss how to reconcile various physical assumptions inherent to different kinetic approaches to dilute gases.
\end{abstract}

\section{INTRODUCTION}

During the last several years there has been a noticeable interest in transverse dynamics of spin-polarized or multicomponent gases, ${ }^{1-11}$ often with an emphasis on quantum gases such as spin-polarized hydrogen or helium (see also review 12 and references therein). By transverse dynamics we mean the dynamics of off-diagonal (mixed) components of the single-particle density matrix, $\widehat{n}$. In the case of spin-polarized gases, the mixed spin component of the density matrix, $n_{\uparrow \downarrow}$, describes the dynamics of the transverse (to the quantization direction) components of magnetization, $n_{\uparrow \downarrow}(\mathbf{p})=m_{x}(\mathbf{p})+i m_{y}(\mathbf{p})$, hence the term "transverse dynamics." In this case our results cover spin dynamics experiments including spin diffusion, spin echo, spin-wave resonances, etc. Generally, the off-diagonal components $n_{\alpha \beta}(\mathbf{p})$ of the density matrix for particles with internal states describe particle oscillations between the internal states $\alpha \leftrightarrow \beta$. Though the results of this paper can be applied to particles with arbitrary internal states, our primary objective is the description of spin-polarized gases. As the only restriction we will assume that the particle interaction should not depend on internal states of particles; in the case of spin states this means that nonexchange interaction forces, such as magnetic dipole-dipole interaction, are weak. This allows one to separate longitudinal and transverse processes.

The behavior of the off-diagonal elements of the density matrix is described by the off-diagonal component of the matrix kinetic (transport) equation. Some insignificant difference between kinetic equations, which are derived by different methods, is caused more by the lack of a rigorous closed procedure of the derivation of the (Boltzmann) transport equation than by any peculiarities of the physical systems. Here we will not go into this subject, and will try to keep the discussion of transverse dynamics at the level where different derivations can still be reconciled.

At high temperatures, when the particle distribution function obeys Boltzmann statistics, the derivation of the Boltzmann kinetic equation was studied rather thoroughly. In the case of particles with internal states the Boltzmann equation is sometimes called the WaldmannSnider equation. Note, however, that the standard formulation of the Waldmann-Snider equation does not fully reflect the fact that quantum particles are identical and treat them as classically identical $;{ }^{9}$ this is important for transverse dynamics and is mostly irrelevant for longitudinal processes. Even in our very limited context, it is impossible to give a meaningful review of the vast literature on the Boltzmann equation; see, e.g., Refs. 13-19 and references in review. ${ }^{20}$

At low temperatures $T \ll T_{F}$, when the particles have a degenerate Fermi distribution, one can use the phenomenological Landau kinetic equation for Fermi liquids. This equation was analyzed by $\mathrm{Silin}^{21}$ and Leggett ${ }^{22}$ for transverse processes at low spin polarization. The Landau-Silin-Leggett equation can be derived microscopically on the basis of the microscopic theory of Fermi liquids; ${ }^{23-26}$ for applications to spin-polarized gases see 
reviews 1 and 12 .

The above high-temperature and low-temperature kinetic approaches can easily be reconciled for longitudinal dynamics in gases, for example, in thermodynamics. For transverse processes, this was possible, so far, only for gases with low spin polarizations. In these cases the microscopic Landau theory ${ }^{23-26}$ provides explicit links between the interaction function and collision integral for phenomenological quasiparticles and the exact particle vertex function. Then all important quasiparticle interaction terms for dilute Fermi systems assume exactly the same form as in the classical kinetic equation which is usually written via the scattering $T$ matrix. As a result, one can write a general kinetic equation for dilute systems at arbitrary temperatures which coincides with the Landau kinetic equation for phenomenological quasiparticles at low temperatures and with the classical kinetic equation at high temperatures. Within this approach, the dressing effects for (quasi)particles become weaker with increasing temperature. Such a uniform kinetic (or thermodynamic) description at arbitrary temperatures in terms of "statistical quasiparticles" is quite well known. ${ }^{27-31}$

The description of transverse dynamics in highly polarized Fermi gases is much less clear. A uniform quasiparticle approach has not been developed despite the existence of different (diagrammatic) methods for derivation of the kinetic equations that are valid at all temperatures. (For application of these methods to spin-polarized quantum gases see Refs. $1,3,5,8,10-12,32$.) The problems are associated less with an extension of the quasiparticle approach to higher temperatures than with a limited applicability of the microscopic and phenomenological Landau theories to transverse dynamics in highly polarized Fermi liquids even at low temperature. The Fermi-liquid theory for transverse processes exhibits several anomalies that do not exist for longitudinal processes and are suppressed at low spin polarizations.

It has been known starting from the first relevant publications in this field ${ }^{21,22,33}$ that a straightforward application of the Landau theory to transverse dynamics at high polarization is questionable because of unavoidable integration of the (quasi)particle distribution function between widely separated Fermi surfaces for spinup and spin-down particles, i.e., effectively, deep into the Fermi sea. Because of strong attenuation of singleparticle states away from the Fermi surface, such an integration is generally forbidden in the Fermi-liquid theory. Though this problem was mentioned in literature from time to time (see, e.g., Refs. 34 and 35 and references therein), a consistent microscopic theory of transverse dynamics in highly polarized Fermi liquids was missing till recently. ${ }^{11,36}$

An experimental manifestation of this problem is a strong anisotropy of spin diffusion and relaxation, namely, a profound difference between spin diffusion coefficients for longitudinal and transverse components of magnetization, $D_{\|}$and $D_{\perp}$, and between diffusion relaxation times $\tau_{\|}$and $\tau_{\perp}$. (Note that this has nothing in common with the well-known difference between relaxation times $T_{1}$ and $T_{2}$ in magnetic systems; both relax- ation times $\tau_{\|}$and $\tau_{\perp}$ have an exchange origin and characterize diffusion of longitudinal and transverse components of magnetization.) What is even more interesting, the transverse kinetic coefficients $D_{\perp}$ and $\tau_{\perp}$ do not increase with decreasing temperature, as all other kinetic coefficients in Fermi liquids, as $1 / T^{2}$, but saturate and remain finite (together with the attenuation of spin waves) even at $T=0$. This anomaly of transverse dynamics in polarized Fermi liquids was predicted by one of the authors on the basis of general symmetry and phase space arguments $^{5}$ (see also review 12), and confirmed later by direct kinetic calculations for polarized Fermi gases $8,10,11$ and, recently, in experiments with spin-polarized ${ }^{3} \mathrm{He}$ ${ }^{4} \mathrm{He}$ mixtures ${ }^{37}$ and pure ${ }^{3} \mathrm{He}^{38}$

Because of this saturation, any phenomenological description in terms of statistical quasiparticles requires an explanation of what happens at higher temperatures to the terms responsible for the zero-temperature attenuation. The existing kinetic description of spin-polarized gases in the Boltzmann temperature region does not exhibit any signs of anomalous dissipative terms.

Zero-temperature attenuation is not the only major difference between the transverse and longitudinal dynamics in polarized Fermi liquids. However, other differences are more technical in nature. The anomalies of transverse dynamics are caused not only by attenuation of single-particle states between the spin-up and spin-down Fermi spheres, but also by a strong spin-upspin-down asymmetry. This effect is similar to the wellknown particle-hole asymmetry away from the Fermi surface: wide separation between spin-up and spin-down Fermi spheres makes the molecular fields and dressing effects for spin-ups and spin-downs distinctly different. The spin-up-spin-down asymmetry means that the transition from the microscopic equation for the transverse component of the Green's function $G_{\uparrow \downarrow}(\omega, \mathbf{p} ; t, \mathbf{r})$ in the four-dimensional (4D) momentum space $\mathbf{P}=(\omega, \mathbf{p})$ to the transverse distribution function $n_{\uparrow \downarrow}(\mathbf{p} ; t, \mathbf{r})$ in the 3D momentum space involves two energy shells $\omega=$ $\epsilon_{\uparrow \uparrow}(\mathbf{p}-\mathbf{k} / 2)$ and $\omega=\epsilon_{\downarrow \downarrow}(\mathbf{p}+\mathbf{k} / 2)$ with distinctly different properties. These two energy shells cannot be reduced to each other by transition to the rotating reference frame except for the case of homogeneous precession $\mathbf{k}=0$. The presence of two different energy shells results in a split of phenomenological equation of transverse dynamics into two different equations (for details, see the first Ref. 11).

Note that the seemingly similar doubling of the number of longitudinal equations is quite trivial; it describes a separation of equations for spin-up and spin-down quasiparticles $n_{\uparrow \uparrow}$ and $n_{\downarrow \downarrow}$ coupled via four different spin components of the interaction $f$ function, $f_{\alpha \beta, \gamma \delta}$. In the longitudinal case we have two different microscopic equations which involve the mass operators and Green's functions, $\Sigma_{\uparrow \uparrow} \Sigma_{\downarrow \downarrow}, G_{\uparrow \uparrow}, G_{\downarrow \downarrow}$, and four spin components of the vertex $\widehat{\Gamma}^{(1)}$ with the obvious implication for the quasiparticle equations. In the transverse case there is only one microscopic equation relating $\Sigma_{\uparrow \downarrow}(\mathbf{P}), G_{\uparrow \downarrow}(\mathbf{P})$ and $\Gamma_{\uparrow \downarrow, \downarrow \uparrow}^{(1)}\left(\mathbf{P}, \mathbf{P}^{\prime}\right)$. The doubling of equations in $n_{\uparrow \downarrow}$ involves only one spin component of the Green's func- 
tion, $G_{\uparrow \downarrow}(\mathbf{P})$, and one component of the vertex function, $\Gamma_{\uparrow \downarrow, \downarrow \uparrow}^{(1)}\left(\mathbf{P}, \mathbf{P}^{\prime}\right)$, but taken on (two) different energy shells (i.e., with different energy components of the vectors $\mathbf{P}$ ). This doubling of transverse equations in $n_{\uparrow \downarrow}$ forces one to operate in terms of two kinds of transverse quasiparticles, both of which contribute to the same transverse component of magnetization. These two quasiparticle-like objects correspond generically to $x-y$ projections of slightly tilted spin-up and spin-down quasiparticles, which, because of spin-up-spin-down asymmetry, experience different molecular fields and precess with different frequencies in inhomogeneous conditions. The corresponding unified quasiparticle description, though technically correct, becomes much less appealing (see the end of the first Ref. 11).

Another anomaly of transverse dynamics is even more technical. It is known that the Fermi-liquid function in nonpolarized systems can be expressed either as one of the limits $\Gamma^{\omega}$ of the full vertex, or by an integral equation involving the irreducible vertex $\Gamma^{(1)}{ }^{24}$ The use of these two equivalent expressions for the interaction function at zero polarization or for longitudinal dynamics often seems to be a mathematical exercise. However, the corresponding expression in transverse dynamics can be given at high polarization only as an integral equation in the irreducible vertex and not as any limit of the full vertex function which loses its singularity ${ }^{11}$ (see also comments in Ref. 34). Since by definition, the $T$ matrix is related to the full vertex, the description in terms of the $T$ matrix, which is natural for Boltzmann gases, becomes impossible at low temperatures.

On the other hand, the transverse dynamics in the high-temperature Boltzmann domain does not reveal any such anomalies. Therefore, it is not clear that it is possible to obtain a simple uniform description in terms of quasiparticles and $T$ matrix which would reproduce such an anomalous Fermi-liquid behavior at low temperatures, and would reduce to a standard Boltzmann description at high temperatures. Of course, one can use a diagrammatic expansion, applicable to all temperatures, in higher and higher orders in interaction and/or density, but it is highly unlikely that these cumbersome equations will be as simple and transparent as quasiparticle description available for longitudinal phenomena.

We will show below that it is still possible to have a simple quasiparticle description for all temperatures in terms of the $T$ matrix up to the second order in the interaction with natural simplifications in all limiting cases. At low temperatures, this description will contain all proper mean-field terms and terms responsible for the zero-temperature attenuation, and will regain the Boltzmann form at higher temperatures. The reason is that the doubling of the transverse quasiparticle equation and analytical problems with the transverse vertex function start only from the third-order terms in the kinetic equation (see the second Ref. 11). Technically this is a consequence of the fact that the vertex $\Gamma_{\uparrow \downarrow, \downarrow \uparrow}^{(1)}\left(\mathbf{P}, \mathbf{P}^{\prime}\right)$ in first order is a constant independent of its four-momentum arguments and, therefore, is not sensitive to the polarization shift between the Fermi spheres. As a result, the kinetic equation up to the second order will still have a fairly traditional form, but will already reflect one of the unique peculiarities of transverse dynamics in polarized Fermi liquids, namely, the zero-temperature attenuation.

We will demonstrate that the concept of statistical quasiparticles, i.e., the application of Landau-like formalism to high-temperature gases, is viable for transverse dynamics in a very wide range of parameters. We will give explicit microscopic expressions for the Landau interaction function and collision integral for statistical quasiparticles via the scattering $T$ matrix that can be used at arbitrary temperatures, from Boltzmann to the degenerate region. The form of the interaction function and collision integral is highly unusual and involves unique pole terms. The temperature-dependent expression for the interaction function becomes especially simple for the so-called quantum gases, i.e., gases of particles with large de Broglie wavelengths. The description of spin dynamics in terms of statistical quasiparticles seems to be more transparent and less cumbersome than within other kinetic approaches.

We can start our analysis from the already existing transverse kinetic equation for arbitrary temperatures ${ }^{7,8}$ obtained by the Kadanoff-Baym method. Unfortunately, this equation is very cumbersome, and it may be easier to derive the same equation anew in a different form than to simplify the already existing equation. We prefer to use a slightly different diagrammatic (Keldysh) method. Our derivation is not "better," but some of the nuances are important, and we feel that our final expression is much more transparent. For obvious reasons, we will not give the details of derivation, but will only outline the procedure by certain focal points in the Appendix.

In the next section we discuss parameters and approximations which are important for the transport equation in different temperature regions with an emphasis on the region of quantum gases. This is necessary because the Boltzmann kinetic theory of gases is based on the density expansion at high temperatures, while the Landau-like approaches are based on the low-temperature expansion at arbitrary density. The obvious place for the overlap is a rarefied "quantum" gas at temperatures that are below the energy of the zero-point motion but can be still higher than the degeneracy temperature $T_{F}$. In Sec. III we will analyze the coherent nondissipative terms in the transport equation for which it is relatively easy to write an equivalent Landau-like interaction function with a natural smooth transition from the Boltzmann to the degenerate region. In Sec. IV we will demonstrate that such a transition persists even for the dissipative terms. This will also show why it is more appropriate to include the unusual terms, responsible for finite attenuation of spin waves and finite transverse spin diffusion at zero temperature, into the interaction function rather than into the collision integral. The results are summarized in Sec. V.

\section{KINETICS OF QUANTUM, DEGENERATE, AND CLASSICAL GASES}

Density $n$ of a rarefied gas is characterized by two dimensionless parameters, $n r_{0}^{3}$ and $n \lambda^{3}$, where $r_{0}$ is the 
interaction radius, and $\lambda$ is the de Broglie wavelength of particles. In dilute gases it is always assumed (with the exception of gases with long-range interaction, e.g., Coulomb gases ) that $n r_{0}^{3} \ll 1$. The expansion in this parameter roughly corresponds to consequent account for two-, three-, and higher-order multiparticle collisions. In practice, one rarely goes beyond the two-particle correlations.

Parameter $n \lambda^{3}$ characterizes the distribution function rather than the density of a gas. This parameter is small for Boltzmann gases, $n \lambda^{3} \ll 1$, and reaches $n \lambda^{3} \sim 1$ at low temperatures when the effects of (Fermi) degeneracy become strong. This means that, at least at low temperatures, the higher-order (density) terms in $n \lambda^{3}$ can be legitimate even if one truncates the main density expansion in $n r_{0}^{3}$ in much lower order. It is convenient to use as independent expansion parameters not these two density parameters, but only one of them (usually, $n r_{0}^{3}$ ), and their ratio [more precisely, $r_{0} / \lambda=\left(n r_{0}^{3} / n \lambda^{3}\right)^{1 / 3} \sim p r_{0} / \hbar$, where $p \sim \hbar / \lambda$ is the characteristic momentum of particles]. At low temperatures this ratio is very small, and increases monotonically with increase in temperature.

The ratio $r_{0} / \lambda \sim p r_{0} / \hbar$ depends on the temperature and, therefore, degeneracy of the system. In Boltzmann gases, $T \gg T_{F} \sim \hbar^{2} n^{2 / 3} / m$, the characteristic momentum $p \sim(m T)^{1 / 2}$. Then the ratio $p r_{0} / \hbar \sim m r_{0}^{2} T / \hbar^{2}$ does not depend on density, and may be much larger as well as smaller than 1 . Usually, the characteristic "quantum" energy parameter $\hbar^{2} / m r_{0}^{2}$ does not exceed $1 \mathrm{~K}$, and for Boltzmann gases at not very low temperatures $T \gg \hbar^{2} / m r_{0}^{2}$ and $r_{0} / \lambda \sim p r_{0} / \hbar \gg 1$. At lower temperatures (and, by necessity, at very low densities - the Fermi temperature scales as $n^{2 / 3}$ ) one can reach the "quantum" region $T \ll \hbar^{2} / m r_{0}^{2}$ and $p r_{0} / \hbar \ll 1$ even for "classical" Boltzmann gases $T \gg T_{F} \sim\left(\hbar^{2} / m\right) n^{2 / 3}$.

For degenerate gases the characteristic Fermi momentum $p_{F} \sim \hbar n^{1 / 3}$. Then the parameter $r_{0} / \lambda \sim$ $p_{F} r_{0} / \hbar$ contains the density and is always small, $r_{0} / \lambda \sim$ $\left(n r_{0}^{3}\right)^{1 / 3} \ll 1$. Below we will use the term "quantum gases" for all gases for which $r_{0} / \lambda \sim p r_{0} / \hbar \ll 1$ (or, in other words, the kinetic energy $\hbar^{2} / m \lambda^{2} \ll \hbar^{2} / m r_{0}^{2}$ ), irrespective of whether the distribution function is a classical (Boltzmann) one $n \lambda^{3} \ll 1$, or is degenerate, $n \lambda^{3} \sim 1$. All degenerate gases $\left(T \ll \hbar^{2} / m \lambda^{2} \sim T_{F} \sim \hbar^{2} n^{2 / 3} / m\right)$ are "quantum" as far as these gases are rarefied, $n r_{0}^{3} \ll 1$, while Boltzmann gases are "quantum" only in the temperature range $T_{F} \sim \hbar^{2} n^{2 / 3} / m \ll T \sim \hbar^{2} / m \lambda^{2} \ll$ $\hbar^{2} / m r_{0}^{2}$. The region of "quantum gases" presents a natural transition between the Boltzmann and degenerate temperature regions.

One of the attractions of quantum gases is that they exhibit macroscopic quantum effects which can be unrelated to the quantum degeneracy of the distribution functions. What is more, for quantum gases the interplay between expansions in $n r_{0}^{3} \ll 1$ and $r_{0} / \lambda \ll 1$ is the same, irrespective of quantum degeneracy of a gas, and the transition from degenerate to Boltzmann gases is rather smooth. Actually, for quantum gases the degeneracy of the distribution function plays the secondary role with respect to quantum effects associated with the large wavelength. Another appealing feature is that these gases are the gases of long-wave $r_{0} / \lambda \ll 1$ (or "slow" $\left.p r_{0} / \hbar \ll 1\right)$ particles. Then the scattering amplitude can be expanded in (small) momentum $p r_{0} / \hbar \ll 1$ and reduces mainly to the $s$-wave scattering with the amplitude $a$ which is almost independent of momentum. The latter feature makes the quantum gases similar to a model system of the identical quantum hard sphere particles, and allows a rather thorough theoretical analysis of all physical phenomena.

The main terms in the collision operator in the kinetic (transport) equation for gases are usually limited to quadratic terms in density $n r_{0}^{3}$ and second order in the interaction (i.e., scattering $T$ matrix). The reason for the first limitation is obvious; the second is more subtle.

For degenerate rarefied gases even the truncated "main" terms in density $n r_{0}^{3} \ll 1$ can include certain higher-order density terms in $p_{F} r_{0} / \hbar \sim\left(n r_{0}^{3}\right)^{1 / 3} \gg n r_{0}^{3}$. On the other hand, the momentum expansion of the vertex function (or $T$ matrix) in $p^{2}$ is, simultaneously, its density expansion since $p_{F} r_{0} / \hbar \sim\left(n r_{0}^{3}\right)^{1 / 3}$. This means that one can consider only the first two-momentum terms, $p_{F} r_{0} / \hbar \sim\left(n r_{0}^{3}\right)^{1 / 3}$ and $\left(p_{F} r_{0} / \hbar\right)^{2} \sim\left(n r_{0}^{3}\right)^{2 / 3}$, in the vertex before having to add the next term in $n r_{0}^{3}$. After that, an addition of extra terms in $p_{F} r_{0} / \hbar$ would require an addition of an extra term in $n r_{0}^{3}$ and is practically impossible. This limits the legitimate contributions to the vertex only to the $s$-wave scattering amplitude (under certain conditions, the $p$-wave scattering amplitude can also be included). The inclusion of the $p$-wave and higher scattering channels into the vertex, which involves higher-order terms in $p_{F} r_{0} / \hbar \sim\left(n r_{0}^{3}\right)^{1 / 3}$, would be beyond the accuracy of a rigorous density expansion for degenerate gases with two-particle collisions. Of course, such terms are highly desirable when one wants to get a more heuristic description of denser systems.

For Boltzmann gases, the secondary expansion in $n \lambda^{3}$ is forbidden at high temperatures, when $n \lambda^{3} \ll n r_{0}^{3}$. It should be considered only at lower temperatures, in the quantum region $r_{0} / \lambda \ll 1$. This means that usually only the single (first) density term is legitimate in the frame of two-particle collisions. However, for Boltzmann gases the characteristic momenta depend only on temperature and do not depend on density. Therefore, for Boltzmann gases the momentum expansion of the $T$ matrix in $p r_{0} / \hbar$ has nothing to do with density expansion, and one can use the exact expression for the $T$ matrix, including all higher scattering channels, even when considering the lowest order in density $n r_{0}^{3}$.

For quantum gases, both expansions in $n r_{0}^{3}$ and $r_{0} / \lambda$ can be performed simultaneously, irrespective of the quantum degeneracy of the gas. This fact has been noticed relatively late, mostly in the context of study of lowtemperature, spin-polarized quantum gases with large macroscopic quantum effects in spin dynamics. ${ }^{1-5,12}$ However, the restriction on the use of the expression for the $T$ matrix depends on degeneracy of the quantum gas.

This difference between degenerate, quantum, and 
Boltzmann gases should be taken into account when analyzing the kinetic equation in the frame of two-particle collisions in different temperature regions. Though the kinetic equation, which is derived in the Appendix, is seemingly the same at all temperatures, the approximation used in the derivation (the truncation of diagrammatic expansion) means different accuracy for different temperature regions. At low temperatures, the absence of higher-order diagrams means that one should disregard all higher-order momentum (density) contributions to the $T$ matrix (basically, all the contributions beyond the $s$-wave scattering). At high temperatures, on the contrary, one can use an exact expression for the $T$ matrix, but should disregard higher-order density terms in the molecular field and collision integral.

Below we are interested in the derivation of a transverse kinetic equation of a Fermi-liquid type which is valid in a wide temperature region. This is equivalent to the description in terms of statistical quasiparticles $^{27,28,30}$ in transverse kinetics. We will get explicit expressions for the quasiparticle interaction function $\widehat{f}\left(\mathbf{p}, \mathbf{p}^{\prime}\right)$ and collision integral $\mathcal{L}\{\widehat{n}\}$, which are applicable at arbitrary temperatures, including the temperatures well above the quantum domain. This kinetic equation should have a Fermi-liquid form

$$
\begin{aligned}
& \partial_{t} \widehat{n}+(i / \hbar)[\widehat{n}, \widehat{\epsilon}]=\widehat{\mathcal{L}}\{\widehat{n}\} \\
& \widehat{\epsilon}(\mathbf{p}, \mathbf{r})=\widehat{\epsilon}_{0}+\int \widehat{f}\left(\mathbf{p}, \mathbf{p}_{1}\right) \delta \widehat{n}\left(\mathbf{p}_{1}, \mathbf{r}\right) d^{3} p_{1} /(2 \pi \hbar)^{3} .
\end{aligned}
$$

What is more, we will see that the interaction function has an imaginary part (only for transverse processes) which gives a new interpretation for the zero-temperature attenuation.

We want to add the following comment. In the narrow sense, the interaction expansion is an expansion in the interaction potential, $\widehat{U}$. There are certain reasons why the kinetic equation should always be expressed not via the interaction energy, $\widehat{U}$, but via the scattering $T$ matrix, $\widehat{T}$ (or the vertex function $\widehat{\Gamma}$ ). These two operators are related to each other by the Lippmann-Schwinger equation,

$$
\widehat{T}=-\widehat{U}-\widehat{U} \widehat{G} \widehat{T},
$$

where $\widehat{G}$ is the Green's function. Therefore, if one wants to express the results, in the spirit of the kinetic theory, through $\widehat{T}$ rather than through $\widehat{U}$, one should substitute $\widehat{U}$ by the series $\widehat{U}=-\widehat{T}+\widehat{T} \widehat{G} \widehat{T}-\widehat{T} \widehat{G} \widehat{T} \widehat{G} \widehat{T}+\cdots$. As we explained in the Introduction, we are interested only in the terms up to the second order in $\widehat{T}$.

\section{MOLECULAR FIELD AND INTERACTION FUNCTION}

We will start from transverse kinetics in the first order in the interaction $T$ matrix $\widehat{T}$. The reason is twofold. First, such an equation presents the main terms of the kinetic equation (1) for quantum (Boltzmann and degenerate) gases or gases with weak interaction. With this accuracy the analogy between the Boltzmann and Landau kinetic equations is very transparent.

In the first order in interaction the attenuation is absent (the attenuation has a definite sign and, therefore, is at least quadratic in interaction). Then all the interaction effects reduce to coherent molecular field, and one should disregard the collision integral in the right-hand side (rhs) of the kinetic equation. Of course, the result is known beforehand: the molecular field in the linear approximation is equal to the forward scattering amplitude, and one can easily express the interaction function $\widehat{f}$ through the $T$ matrix $\widehat{T}$. According to Eq. (A12), the transverse kinetic equation in the first order in the interaction is

$$
\begin{aligned}
\partial_{t} n_{\downarrow \uparrow}(\mathbf{p})+ & \frac{i}{\hbar}\left[\widehat{\epsilon}_{0}, \widehat{n}\right]_{\downarrow \uparrow}(\mathbf{p}) \\
= & \frac{i}{\hbar}(2 \pi \hbar)^{-3} \int T\left(\mathbf{p}_{1}-\mathbf{p}\right) \\
& \times\left\{m\left(\mathbf{p}_{1}\right) n_{\downarrow \uparrow}(\mathbf{p})-m(\mathbf{p}) n_{\downarrow \uparrow}\left(\mathbf{p}_{1}\right)\right\} d^{3} p_{1} .
\end{aligned}
$$

Since there is no attenuation in the first order, we could also easily get the same equation using the Fermi liquid approach by calculating the variational derivative of the full energy

$$
E=E_{0}+\sum_{\mathbf{p}, \mathbf{p}_{1}, \sigma, \sigma_{1}} U_{\sigma \sigma_{1}}\left(\mathbf{p}-\mathbf{p}_{1}\right) n_{\sigma}(\mathbf{p}) n_{\sigma_{1}}\left(\mathbf{p}_{1}\right)
$$

over the transverse distribution functions and substituting the corresponding transverse $f$ function,

$$
\begin{aligned}
f_{\sigma \sigma_{1}}^{(1)}\left(\mathbf{p}, \mathbf{p}_{1}\right) & \equiv \frac{\delta^{2} E}{\delta n_{\sigma}(\mathbf{p}) \delta n_{\sigma_{1}}\left(\mathbf{p}_{1}\right)} \\
& =T\left(\mathbf{p}-\mathbf{p}_{1}\right)\left(1-\vec{\sigma} \cdot \vec{\sigma}_{1}\right)
\end{aligned}
$$

into the Fermi-liquid kinetic equation (1) [in Eq. 5 we took into account that in the first order in the interaction the scattering $T$ matrix (2) coincides with the interaction operator $\left.U_{\sigma \sigma_{1}}(\mathbf{p})=U(\mathbf{p})\left(1-\vec{\sigma} \cdot \vec{\sigma}_{1}\right)\right]$.

This confirms the trivial fact that the phenomenological Fermi-liquid-like approach of statistical quasiparticles works perfectly in the first order in interaction at arbitrary temperatures. Equation (3) has been derived, in different forms, by many authors (see, e.g., Refs. 1, 3-5, 7,12 and references therein). This equation has sufficient accuracy either when the interaction is weak, or for quantum gases for which $r_{0} / \lambda \ll 1$ and all the dissipative and other second-order terms are small in $r_{0} / \lambda \ll 1$.

One should keep in mind that, according to the preceding section, one should always substitute the scattering amplitude in Eq. (3) for dilute degenerate gases by a constant $-a$ (i.e., by the $s$-wave scattering length),

$$
T\left(\mathbf{p}, \mathbf{p}_{1}\right) \rightarrow-\frac{4 \pi \hbar^{2}}{M} a, \quad a=\frac{M}{4 \pi \hbar^{2}} \int U(\mathbf{r}) d^{3} r
$$

where $M=m / 2$ is the reduced mass of particles with the mass $m$. The full function $T\left(\mathbf{p}, \mathbf{p}_{1}\right)$ differs from the scattering length $a$ by the terms which are equivalent, at 
low temperatures $T \ll T_{F}$, to the higher-order terms in density $p_{F} r_{0} / \hbar \sim\left(n r_{0}^{3}\right)^{1 / 3}$. These higher-order density terms are effectively smaller than the already neglected terms of the higher order in the interaction (see, e.g., Refs. 1 and 12), and ought to be neglected as well. At higher temperatures, for dilute nondegenerate gases, the difference between the exact scattering amplitude and the scattering length is not necessarily smaller than the neglected higher-order interaction terms; in this case the use of the exact scattering amplitude in Eq. (3) is quite legitimate and, in general, preferable.

In the second order in the interaction, the coherent molecular field in the transverse kinetic equation (A15) at arbitrary temperatures is

$$
\begin{aligned}
L_{\mathrm{coh}}= & \frac{i m}{\hbar} \int \frac{d^{3} p^{\prime}}{(2 \pi \hbar)^{3}} \frac{d^{3} p_{1}}{(2 \pi \hbar)^{3}} P \frac{1}{p^{2}+p_{1}^{2}-p^{\prime 2}-\left(\mathbf{p}+\mathbf{p}_{1}-\mathbf{p}^{\prime}\right)^{2}}\left\{T\left(\mathbf{p}_{1}-\mathbf{p}^{\prime}\right) T\left(\mathbf{p}^{\prime}-\mathbf{p}\right) n^{\prime}\left[n_{\uparrow \downarrow}\left(\mathbf{p}_{1}\right) m-m_{1} n_{\uparrow \downarrow}(\mathbf{p})\right]\right. \\
& \left.+\left[T^{2}\left(\mathbf{p}_{1}-\mathbf{p}^{\prime}\right)-T\left(\mathbf{p}_{1}-\mathbf{p}_{1}^{\prime}\right) T\left(\mathbf{p}^{\prime}-\mathbf{p}_{1}\right)\right]\left(n^{\prime}-n_{1}\right)\left[n_{\uparrow \downarrow}\left(\mathbf{p}_{1}^{\prime}\right) m-n_{\uparrow \downarrow}(\mathbf{p}) m\left(\mathbf{p}_{1}^{\prime}\right)\right]\right\}
\end{aligned}
$$

where $\mathbf{p}_{1}^{\prime}=\mathbf{p}+\mathbf{p}_{1}-\mathbf{p}^{\prime}, n(\mathbf{p}) \equiv n_{\uparrow \uparrow}^{(0)}(\mathbf{p})+n_{\downarrow \downarrow}^{(\mathbf{0})}(\mathbf{p})$, and $m(\mathbf{p}) \equiv n_{\uparrow \uparrow}^{(0)}(\mathbf{p})-n_{\downarrow \downarrow}^{(0)}(\mathbf{p}), n_{i} \equiv n\left(\mathbf{p}_{i}\right), m_{i} \equiv m\left(\mathbf{p}_{i}\right)$.

This is the so-called $I_{2}$ term which has been repeatedly discussed in the literature. At first, the term with $P \frac{1}{\epsilon+\epsilon_{1}-\epsilon^{\prime}-\epsilon_{1}^{\prime}}$ in molecular field was suggested in Ref. 39 on the basis of the perturbation expansion in $\widehat{U}$. However, the $I_{2}$ term in Ref. 39 had a lower order in density than the third-order term (7). It soon became clear ${ }^{40,32}$ that the second-order density $I_{2}$ term suggested in Ref. 39 was erroneous and should disappear from the kinetic equation as a result of the transformation (renormalization) from the interaction $\widehat{U}$ to the $\widehat{T}$ matrix (see the relevant discussion in the Appendix). The silent consensus was that all the terms with $P \frac{1}{\epsilon+\epsilon_{1}-\epsilon^{\prime}-\epsilon_{1}^{\prime}}$ always disappear from the transverse transport equation, at least for Boltzmann gases, though this has never been checked for higher-order density/perturbation terms.

As we see, the third-order $I_{2}$ term (7) exists and has the same form at all temperatures. This term contains the product of three distribution functions and should be used at high temperatures very cautiously since the standard kinetic equations for Boltzmann gases contains the product of maximum two distributions. For the same reason, this term does not show up in standard derivations of the high-temperature Boltzmann equation. Though this term is legitimate, it should often be dropped so far as other (neglected) terms of the third order in the distribution, like the terms in Refs. 41 and 35, may be important. Generally, this $I_{2}$ term can be used in the classical Boltzmann temperature range only if its $P \frac{1}{\epsilon}$ structure is significant and unique. At lower temperatures, the unrestricted use of this term does not cause any problems.

The molecular-field terms (7) in the kinetic equation can be rewritten in the form of Landau interaction function $f_{\sigma \sigma_{1}}^{(2)}\left(\mathbf{p}, \mathbf{p}_{1}\right)$. Comparison of Eqs. (3) and (7) shows that the equivalent expression for the transverse $\left(\vec{\sigma} \cdot \vec{\sigma}_{1}\right)$ component of the Landau function in the lhs of the kinetic equation can be reduced, after some algebra, to the form

$$
\begin{aligned}
\operatorname{Re} f_{\sigma \sigma_{1}}^{(2)}\left(\mathbf{p}, \mathbf{p}_{1}\right)= & \frac{1}{4} \vec{\sigma} \cdot \vec{\sigma}_{1} \operatorname{Re} \int \frac{d^{3} p^{\prime}}{(2 \pi \hbar)^{3}}\left\{T\left(\mathbf{p}_{1}-\mathbf{p}^{\prime}\right) T\left(\mathbf{p}_{1}^{\prime}-\mathbf{p}_{1}\right) \frac{n^{\prime}}{\epsilon+\epsilon_{1}-\epsilon^{\prime}-\epsilon_{1}^{\prime}-i 0 \operatorname{sgn}\left(p^{\prime}-p_{\downarrow}\right)}\right. \\
& +\left[T^{2}\left(\mathbf{p}_{1}^{\prime}-\mathbf{p}^{\prime}\right)-T\left(\mathbf{p}_{1}^{\prime}-\mathbf{p}_{1}\right) T\left(\mathbf{p}_{1}^{\prime}-\mathbf{p}^{\prime}\right)\right] \\
& \left.\times\left[\frac{n_{\downarrow}^{\prime}-n_{1 \downarrow}^{\prime}}{\epsilon+\epsilon_{1}-\epsilon^{\prime}-\epsilon_{1}^{\prime}-i 0 \operatorname{sgn}\left(p^{\prime}-p_{\downarrow}\right)}+\frac{n_{\uparrow}^{\prime}-n_{1 \uparrow}^{\prime}}{\epsilon+\epsilon_{1}-\epsilon^{\prime}-\epsilon_{1}^{\prime}-i 0 \operatorname{sgn}\left(p^{\prime}-p_{\uparrow}\right)}\right]\right\},
\end{aligned}
$$

where $\mathbf{p}_{1}^{\prime}=\mathbf{p}+\mathbf{p}_{1}-\mathbf{p}^{\prime}$ and $\epsilon_{i}=\epsilon\left(\mathbf{p}_{i}\right)=p_{i}^{2} / 2 m$.

An interesting simplification occurs for quantum gases when the $\widehat{T}$ matrix is a constant, Eq. (6), and the last two terms in Eqs. (7) or (8) cancel each other. The remaining, first, term in the interaction function (8) is

$$
\operatorname{Re} f_{\sigma \sigma_{1}}^{(2)}\left(\mathbf{p}, \mathbf{p}_{1}\right)=\frac{32 \pi^{2} a^{2} \hbar^{4}}{m} \operatorname{Re} \sum_{\mathbf{p}^{\prime}} \frac{n\left(\mathbf{p}^{\prime}\right) \vec{\sigma} \cdot \vec{\sigma}_{1}}{p^{2}+p_{1}^{2}-p^{\prime 2}-p_{1}^{\prime 2}-i 0 \operatorname{sgn}\left(p^{\prime}-p_{\downarrow}\right)}
$$

and is exactly the same as the real part of the transverse Landau $f$-function of spin-polarized quantum gases calculated in Refs. 1 and 11.

We want to emphasize that the imaginary (pole) part of this function cannot be recovered by calculating variational derivatives of the energy as in phenomenological Landau theory [cf. Eq. (5)]: the summation over intermediate states for the second-order interaction corrections 
for the energy

$$
E_{n}^{(2)}=\sum_{m}{ }^{\prime} \frac{\left|U_{m n}\right|^{2}}{E_{n}-E_{m}}
$$

excludes the states $E_{n}=E_{m}$, and, therefore, reduces to the real (principal) part of the integral (9). To obtain the imaginary part within the phenomenological approach, one has to insert the factor $i 0$ in the denominator in order to reproduce the whole equations (8) and (9). However, the microscopic calculations for spin-polarized Fermi liquids ${ }^{11}$ give direct justification for the presence of this factor. More detailed comments on the imaginary part of the Landau function (7) will be given in the next section.

The real part of the $f$ function (9), i.e., the principal value of the integral, is equivalent to the following molecular-field term in the transverse component of the transport equation:

$$
\begin{aligned}
L_{\mathrm{coh}}= & \frac{32 \pi^{2} \hbar^{3} a^{2}}{m^{2}} P \sum_{\mathbf{p}^{\prime}, \mathbf{p}_{1}} \frac{1}{\epsilon+\epsilon_{1}-\epsilon^{\prime}-\epsilon_{1}^{\prime}} \\
& \times n^{\prime}\left[n_{\uparrow \downarrow}\left(\mathbf{p}_{1}\right) m-n_{\uparrow \downarrow}(\mathbf{p}) m_{1}\right] .
\end{aligned}
$$

The integrals in the real part of the transverse Landau function (9) were calculated at $T=0$ in Ref. 1:

$$
\operatorname{Re} f^{(2)}=\frac{2 \hbar a^{2}}{m}\left[I^{+}\left(\mathbf{p}, \mathbf{p}_{1}\right)+I^{-}\left(\mathbf{p}, \mathbf{p}_{1}\right)\right] \vec{\sigma} \cdot \vec{\sigma}_{1}
$$

$$
\begin{aligned}
I^{ \pm}\left(\mathbf{p}, \mathbf{p}_{1}\right)= & \frac{q}{4} \ln \frac{p_{ \pm} q+p_{ \pm}^{2}-\mathbf{p} \cdot \mathbf{p}_{1}}{p_{ \pm} q-p_{ \pm}^{2}+\mathbf{p} \cdot \mathbf{p}_{1}}-p_{ \pm} \\
& -\frac{2 p_{ \pm}^{2}-p^{2}-p_{1}^{2}}{4 r} \ln \frac{p_{ \pm} q+p_{ \pm}^{2}+\mathbf{p} \cdot \mathbf{p}_{1}}{p_{ \pm} q-p_{ \pm}^{2} \mathbf{p} \cdot \mathbf{p}_{1}} \\
r= & \left|\mathbf{p}+\mathbf{p}_{1}\right|, \quad q=\left|\mathbf{p}-\mathbf{p}_{1}\right| .
\end{aligned}
$$

The integrals for the imaginary part can be found in Ref. 11.

\section{COLLISION INTEGRAL AND INTERACTION FUNCTION}

In this section we will study the dissipative collision integral. We will demonstrate that at $T=0$ the collision integral can be represented as an imaginary (pole) part of the interaction function from Sec. III. This provides a new interpretation for the zero-temperature attenuation in transverse spin dynamics ${ }^{11,36-38}$ as an imaginary pole term in the interaction function of statistical quasiparticles. This will also emphasize that the statistical quasiparticles in transverse spin dynamics of spin-polarized gases have a finite lifetime even at $T=0$. Then it is only natural to rewrite the full collision integral at finite temperatures as a combination of a temperaturedependent imaginary (pole) contribution from the interaction function and the remaining terms, which should be interpreted as a collision integral of statistical quasiparticles.

According to Eq. (A17) of the Appendix, the dissipative collision integral in the second order in the interaction has the form

$$
\begin{aligned}
L_{\mathrm{coll}}= & \frac{\pi}{2 \hbar} \int \frac{d^{3} p_{1}}{(2 \pi \hbar)^{3}} \frac{d^{3} p^{\prime}}{(2 \pi \hbar)^{3}} \delta\left(\epsilon+\epsilon_{1}-\epsilon^{\prime}-\epsilon_{1}^{\prime}\right)\left\{T ( \mathbf { p } _ { 1 } - \mathbf { p } ^ { \prime } ) T ( \mathbf { p } _ { 1 } ^ { \prime } - \mathbf { p } _ { 1 } ) \left\{\left(1-n^{\prime}\right)\left[n_{\uparrow \downarrow}\left(\mathbf{p}_{1}\right) n-n_{\uparrow \downarrow}(\mathbf{p}) n_{1}\right]\right.\right. \\
& \left.+\frac{1}{2}\left[n_{\uparrow \downarrow}\left(\mathbf{p}_{1}\right)-n_{\uparrow \downarrow}(\mathbf{p})\right]\left(n^{\prime} n_{1}^{\prime}-m^{\prime} m_{1}^{\prime}\right)\right\} \\
& \left.+\left[T^{2}\left(\mathbf{p}_{1}-\mathbf{p}^{\prime}\right)-T\left(\mathbf{p}_{1}-\mathbf{p}^{\prime}\right) T\left(\mathbf{p}_{1}^{\prime}-\mathbf{p}_{1}\right)\right]\left[\begin{array}{c}
n_{\uparrow \downarrow}\left(\mathbf{p}_{1}^{\prime}\right)\left[n n_{1}+n^{\prime}(2-n)-n^{\prime} n_{1}-m^{\prime} m_{1}\right] \\
-n_{\uparrow \downarrow}(\mathbf{p})\left[n^{\prime} n_{1}^{\prime}+n_{1}\left(2-n_{1}^{\prime}\right)-n^{\prime} n_{1}-m^{\prime} m_{1}\right]
\end{array}\right]\right\} .
\end{aligned}
$$

This equation is too cumbersome, and we will start from its equivalent in the $s$-wave scattering approximation for the quantum gases:

$$
\begin{aligned}
L_{\mathrm{coll}}= & \frac{32 \pi^{3} \hbar^{3} a^{2}}{m^{2}} \int \frac{d^{3} p_{1}}{(2 \pi \hbar)^{3}} \frac{d^{3} p^{\prime}}{(2 \pi \hbar)^{3}} \delta\left(\epsilon+\epsilon_{1}-\epsilon^{\prime}-\epsilon_{1}^{\prime}\right) \\
& \times\left\{\left(1-n^{\prime}\right)\left[n_{\uparrow \downarrow}\left(\mathbf{p}_{1}\right) n-n_{\uparrow \downarrow}(\mathbf{p}) n_{1}\right]+\frac{1}{2}\left[n_{\uparrow \downarrow}\left(\mathbf{p}_{1}\right)-n_{\uparrow \downarrow}(\mathbf{p})\right]\left(n^{\prime} n_{1}^{\prime}-m^{\prime} m_{1}^{\prime}\right)\right\} .
\end{aligned}
$$

This equation can, in turn, be rewritten as

$$
\begin{aligned}
L_{\mathrm{coll}}= & \frac{32 \pi^{3} \hbar^{3} a^{2}}{m^{2}} \int \frac{d^{3} p_{1}}{(2 \pi \hbar)^{3}} \frac{d^{3} p^{\prime}}{(2 \pi \hbar)^{3}} \delta\left(\epsilon+\epsilon_{1}-\epsilon^{\prime}-\epsilon_{1}^{\prime}\right)\left\{n_{\uparrow \downarrow}\left(\mathbf{p}_{1}\right)\left[\left(1-n_{\downarrow \downarrow}^{\prime}-n_{1 \uparrow \uparrow}^{\prime}\right) n+2 n_{\uparrow \uparrow}^{\prime} n_{1 \downarrow \downarrow}^{\prime}\right]\right. \\
& \left.-n_{\uparrow \downarrow}(\mathbf{p})\left[\left(1-n_{\downarrow \downarrow}^{\prime}-n_{1 \uparrow \uparrow}^{\prime}\right) n_{1}+2 n_{\uparrow \uparrow}^{\prime} n_{1 \downarrow \downarrow}^{\prime}\right]\right\} .
\end{aligned}
$$

The transverse density $n_{\downarrow \uparrow}$ is a trace of the operator $a_{\downarrow \mathbf{p}}^{\dagger} a_{\uparrow \mathbf{p}^{\prime}}$. Obviously, this operator is nonzero at $T=0$ only in the belt between spin-up and spin-down Fermi spheres, $p_{\downarrow} \leq p \leq p_{\uparrow}$. Therefore, at $T=0$ one should consider the transport equation only inside this belt [microscopic equations reproduce this condition automatically by having 
$\theta\left(p-p_{\uparrow}\right)-\theta\left(p-p_{\downarrow}\right)$ in all important integrands $\left.{ }^{11}\right]$. What is more, in this belt at $T=0$ the equilibrium distribution functions $n=n_{1}=1, m=m_{1}=1$, and the collision integral,

$$
L_{\mathrm{coll}}(T=0)=\frac{32 \pi^{3} \hbar^{3} a^{2}}{m^{2}} \int \frac{d^{3} p_{1}}{(2 \pi \hbar)^{3}} \frac{d^{3} p^{\prime}}{(2 \pi \hbar)^{3}} \delta\left(\epsilon+\epsilon_{1}-\epsilon^{\prime}-\epsilon_{1}^{\prime}\right)\left[n_{\uparrow \downarrow}\left(\mathbf{p}_{1}\right)-n_{\uparrow \downarrow}(\mathbf{p})\right]\left[\left(1-n_{\downarrow \downarrow}^{\prime}\right)\left(1-n_{1 \uparrow \uparrow}^{\prime}\right)+n_{\downarrow \downarrow}^{\prime} n_{1 \uparrow \uparrow}^{\prime}\right]
$$

can be written as

$$
\begin{aligned}
L_{\mathrm{coll}}(T=0)= & \frac{32 \pi^{2} \hbar^{3} a^{2}}{m^{2}} \operatorname{Im} \int \frac{d^{3} p_{1}}{(2 \pi \hbar)^{3}} \frac{d^{3} p^{\prime}}{(2 \pi \hbar)^{3}}\left[n_{\uparrow \downarrow}\left(\mathbf{p}_{1}\right)-n_{\uparrow \downarrow}(\mathbf{p})\right] \\
& \times\left[\left(1-n_{\downarrow \downarrow}^{\prime}\right)\left(1-n_{1 \uparrow \uparrow}^{\prime}\right)+n_{\downarrow \downarrow}^{\prime} n_{1 \uparrow \uparrow}^{\prime}\right] /\left(\epsilon+\epsilon_{1}-\epsilon^{\prime}-\epsilon_{1}^{\prime}-i 0\right) .
\end{aligned}
$$

Of course, the last equation is completely equivalent to the use of the Landau interaction function $f_{\sigma \sigma^{\prime}}\left(\mathbf{p}, \mathbf{p}_{1}\right)$,

$$
L_{\text {coll }}=\frac{i}{\hbar} \int \frac{d^{3} p_{1}}{(2 \pi \hbar)^{3}} \operatorname{Im} f^{(2)}\left(\mathbf{p}, \mathbf{p}_{1}\right)\left[n_{\uparrow \downarrow}\left(\mathbf{p}_{1}\right) m-n_{\uparrow \downarrow}(\mathbf{p}) m_{1}\right]
$$

if the interaction function has the form

$$
\operatorname{Im} f_{\sigma \sigma_{1}}^{(2)}\left(\mathbf{p}, \mathbf{p}_{1}\right)=\frac{32 \pi^{2} a^{2} \hbar^{4}}{m} \operatorname{Im} \int \frac{d^{3} p^{\prime}}{(2 \pi \hbar)^{3}} \frac{\left(n_{\downarrow \downarrow}^{\prime}+n_{1 \uparrow \uparrow}^{\prime}-1\right) \vec{\sigma} \cdot \vec{\sigma}_{1}}{p^{2}+p_{1}^{2}-p^{\prime 2}-p_{1}^{\prime 2}-i 0 \operatorname{sgn}\left(p^{\prime}-p_{\downarrow}\right)}
$$

[the imaginary pole term $i 0 \operatorname{sgn}\left(p^{\prime}-p_{\downarrow}\right)$ in the denominator is equivalent to the factor $i \pi\left(1-2 n_{\downarrow \downarrow}^{\prime}\right) \delta\left(p^{2}+p_{1}^{2}-p^{\prime 2}-p_{1}^{\prime 2}\right)$ in the numerator; in order to reproduce Eq. (14) from Eq. (9), one should also symmetrize both equations with the help of the transformation $\left.\mathbf{p}^{\prime} \leftrightarrow \mathbf{p}_{1}^{\prime}\right]$. Bringing together Eqs. (9) and (17), one can finally write the full expression for the transverse part of the Landau interaction function:

$$
f_{\sigma \sigma_{1}}^{(2)}\left(\mathbf{p}, \mathbf{p}_{1}\right)=-\frac{32 \pi^{2} a^{2} \hbar^{4}}{m} \vec{\sigma} \cdot \vec{\sigma}_{1} \int \frac{d^{3} p^{\prime}}{(2 \pi \hbar)^{3}}\left[\frac{1-n_{\downarrow \downarrow}^{\prime}-n_{1 \uparrow \uparrow}^{\prime}}{p^{2}+p_{1}^{2}-p^{\prime 2}-p_{1}^{\prime 2}-i 0 \operatorname{sgn}\left(p^{\prime}-p_{\downarrow}\right)}-P \frac{1}{p^{2}+p_{1}^{2}-p^{2}-p_{1}^{\prime 2}}\right] .
$$

Not surprisingly, this interaction function is completely equivalent to the result of microscopic calculation of the irreducible vertex function in Ref. 11 at $T=0$. Note that the presence of the $P \frac{1}{\epsilon}$ term in Eq. (18) reflects the interaction renormalization in the molecular field (see the Appendix). One can also write this equation in a more symmetric form with the help of the transformation $\mathbf{p}^{\prime} \leftrightarrow \mathbf{p}_{1}^{\prime}$.

Equation (18) for the effective transverse interaction functions in spin-polarized gases is one of the main results of this paper. As it was shown in the preceding section, the real part of this interaction function correctly reproduces all the coherent molecular field at all temperatures. This is not so for the imaginary part of the interaction function (18), which reproduces the correct attenuation only at $T=0$, i.e., the zero-temperature attenuation. This gives us a new interpretation of the zero-temperature attenuation as the imaginary part of the interaction function for quasiparticles. This situation remotely resembles the well-known case of the collisionless Landau damping in plasma when the attenuation is also associated with similar analytical properties of the molecular field.

At finite temperatures there are two sources of temperature-driven attenuation with the $T^{2}$ dependence: the imaginary part of the interaction function (18) [which, at $T \neq 0$, does not have the form of (14) and (15) since $\operatorname{sgn}\left(p^{\prime}-p_{\downarrow}\right)$ is not equal to $1-2 n_{\downarrow \downarrow}^{\prime}(T \neq 0)$ and $\left.n_{\downarrow \downarrow}^{\prime}(T \neq 0) \neq n_{\downarrow \downarrow}^{\prime 2}(T \neq 0)\right]$, and the neglected terms in the collision integral (13) which are zero at $T=0$. All these terms can be written in the form of the effective collision integral for statistical quasiparticles with the interaction function (18):

$$
\begin{aligned}
\mathcal{L}_{\text {coll }}= & \frac{32 \pi^{3} \hbar^{3} a^{2}}{m^{2}} \int \frac{d^{3} p_{1}}{(2 \pi \hbar)^{3}} \frac{d^{3} p^{\prime}}{(2 \pi \hbar)^{3}} \delta\left(\epsilon+\epsilon_{1}-\epsilon^{\prime}-\epsilon_{1}^{\prime}\right)\left[n_{\uparrow \downarrow}\left(\mathbf{p}_{1}\right)(n-1)-n_{\uparrow \downarrow}(\mathbf{p})\left(n_{1}-1\right)\right]\left(1-n^{\prime}\right) \\
& +2\left[n_{\uparrow \downarrow}\left(\mathbf{p}_{1}\right)-n_{\uparrow \downarrow}(\mathbf{p})\right]\left[n_{\uparrow \uparrow}^{\prime}\left(n_{1 \downarrow \downarrow}^{\prime(0)}-n_{1 \downarrow \downarrow}^{\prime}\right)+n_{\downarrow \downarrow}^{\prime(0)}\left(1-n_{\downarrow \downarrow}^{\prime}\right)\right]
\end{aligned}
$$

where the upper index (0) denotes the corresponding distribution at $T=0$. This collision integral is the transverse component for the Fermi-liquid collision operator for quasiparticles with the interaction function (18). Equations (18) and (19) allow one to use the Landau-like kinetic equation (1) in the whole temperature range for quantum gases.

We will also give analogous general expressions for the interaction function and the collision integral for statistical quasiparticles outside the $s$-wave approximation, i.e., via the exact scattering amplitude $T$ (p). The general expression for the interaction function has the form 


$$
\begin{aligned}
f_{\sigma \sigma_{1}}^{(2)}\left(\mathbf{p}, \mathbf{p}_{1}\right)= & -\frac{1}{4} \vec{\sigma} \cdot \vec{\sigma}_{1} \int \frac{d^{3} p^{\prime}}{(2 \pi \hbar)^{3}}\left\{T\left(\mathbf{p}_{1}-\mathbf{p}^{\prime}\right) T\left(\mathbf{p}_{1}^{\prime}-\mathbf{p}_{1}\right)\left[\frac{1-n^{\prime}}{\epsilon+\epsilon_{1}-\epsilon^{\prime}-\epsilon_{1}^{\prime}-i 0 \operatorname{sgn}\left(p^{\prime}-p_{\downarrow}\right)}-P \frac{1}{\epsilon+\epsilon_{1}-\epsilon^{\prime}-\epsilon_{1}^{\prime}}\right]\right. \\
& -\left[T^{2}\left(\mathbf{p}_{1}^{\prime}-\mathbf{p}^{\prime}\right)-T\left(\mathbf{p}_{1}^{\prime}-\mathbf{p}_{1}\right) T\left(\mathbf{p}_{1}^{\prime}-\mathbf{p}^{\prime}\right)\right] \\
& \left.\times\left[\frac{n_{\downarrow \downarrow}^{\prime}-n_{1 \downarrow \downarrow}^{\prime}}{\epsilon+\epsilon_{1}-\epsilon^{\prime}-\epsilon_{1}^{\prime}-i 0 \operatorname{sgn}\left(p^{\prime}-p_{\downarrow}\right)}+\frac{n_{\uparrow \uparrow}^{\prime}-n_{1 \uparrow \uparrow}^{\prime}}{\epsilon+\epsilon_{1}-\epsilon^{\prime}-\epsilon_{1}^{\prime}-i 0 \operatorname{sgn}\left(p^{\prime}-p_{\uparrow}\right)}\right]\right\}
\end{aligned}
$$

and reduces to Eq. (18) when the scattering amplitude is constant and the last term is cancelled out. The corresponding collision integral is very cumbersome:

$$
\begin{aligned}
\mathcal{L}_{\mathrm{coll}}= & \int \frac{d^{3} p_{1}}{(2 \pi \hbar)^{3}} \frac{d^{3} p^{\prime}}{(2 \pi \hbar)^{3}} \delta\left(\epsilon+\epsilon_{1}-\epsilon^{\prime}-\epsilon_{1}^{\prime}\right)\left\{T\left(\mathbf{p}_{1}-\mathbf{p}^{\prime}\right) T\left(\mathbf{p}_{1}^{\prime}-\mathbf{p}_{1}\right)\right\}\left\{\left[n_{\uparrow \downarrow}\left(\mathbf{p}_{1}\right)(n-1)-n_{\uparrow \downarrow}(\mathbf{p})\left(n_{1}-1\right)\right]\left(1-n^{\prime}\right)\right. \\
& \left.+2\left[n_{\uparrow \downarrow}\left(\mathbf{p}_{1}\right)-n_{\uparrow \downarrow}(\mathbf{p})\right]\left[n_{\uparrow \uparrow}^{\prime}\left(n_{1 \downarrow \downarrow}^{\prime(0)}-n_{1 \downarrow \downarrow}^{\prime}\right)+n_{\downarrow \downarrow}^{\prime(0)}\left(1-n_{\downarrow \downarrow}^{\prime}\right)\right]\right\} \\
& +\delta\left(\epsilon+\epsilon_{1}^{\prime}-\epsilon^{\prime}-\epsilon_{1}\right)\left[T^{2}\left(\mathbf{p}_{1}^{\prime}-\mathbf{p}^{\prime}\right)-T\left(\mathbf{p}_{1}^{\prime}-\mathbf{p}_{1}\right) T\left(\mathbf{p}_{1}^{\prime}-\mathbf{p}^{\prime}\right)\right] \\
& \times\left\{2\left[n_{\uparrow \downarrow}(\mathbf{p})-n_{\uparrow \downarrow}\left(\mathbf{p}_{1}\right)\right]\left[n_{1 \uparrow \uparrow}^{\prime}\left(n_{\uparrow \uparrow}^{\prime}-n_{\uparrow \uparrow}^{\prime(0)}\right)+n_{1 \downarrow \downarrow}^{\prime}\left(n_{\downarrow \downarrow}^{\prime}-n_{\downarrow \downarrow}^{\prime(0)}\right)\right]\right. \\
& +n_{\uparrow \downarrow}\left(\mathbf{p}_{1}\right)\left[\left(n_{1}^{\prime}-n^{\prime}\right)(n-1)+2 n_{\uparrow \uparrow}^{\prime}\left(1-n_{\uparrow \uparrow}^{\prime(0)}\right)+2 n_{\downarrow \downarrow}^{\prime}\left(1-n_{\downarrow \downarrow}^{\prime(0)}\right)\right] \\
& \left.-n_{\uparrow \downarrow}(\mathbf{p})\left[\left(n^{\prime}-n_{1}^{\prime}\right)\left(n_{1}-1\right)+2 n_{\uparrow \uparrow}^{\prime}\left(1-n_{\uparrow \uparrow}^{\prime(0)}\right)+2 n_{\downarrow \downarrow}^{\prime}\left(1-n_{\downarrow \downarrow}^{\prime(0)}\right)\right]\right\} .
\end{aligned}
$$

The expressions (18)-(21) constitute the main results of this paper. We want to repeat that in the lowtemperature "quantum" region one should always use Eqs. (18) and (19) rather than "exact" Eqs. (20) and (21).

\section{DISCUSSION. BEYOND THE SECOND ORDER}

The combination of the interaction function (20) with the collision integral (21) [or (18) with (19)] allows one to extend the Fermi-liquid description of transverse dynamics (1) in spin-polarized gases to all temperatures. These equations give the justification for the use of the description in terms of statistical quasiparticles in transverse spin dynamics. In these terms, the zero-temperature attenuation and the $I_{2}$ terms are simply the imaginary (pole) and real (principal) parts of the same quasiparticle interaction function which, at $T=0$, coincides with the exact microscopic expression for the irreducible vertex part.

The existence of different types of $T^{2}$ temperature terms in attenuation, namely, the temperature dependence of the imaginary part of the interaction function (18) and (20) and two types of terms in the collision integral (19) and (21), can explain the existence of two different regimes in the temperature dependence of the transverse spin-diffusion coefficient described recently in computations. $^{42}$

Another important conclusion is that the separation of the terms in the kinetic equation into commutator ("molecular field") and anticommutator ("collision integral"), as it is usually done (and is done in the Appendix), is not unique and is somewhat arbitrary. Above we demonstrated that the nonvanishing at $T=0$ part of the anticommutator can be effectively reduced to the (imaginary) commutator and included in the molecular field as its imaginary (pole) part. Such a possibility of transfer of certain terms between the commutator and anticommutator (or coherent and collision) parts of the kinetic equation can often lead to a terminological misunderstanding and obscure the physics, especially in more convoluted situations. Above we included in the "molecular field" (interaction function $f^{(2)}$ ) all the terms, including the imaginary ones, which do not vanish at $T=0$. These terms in the kinetic equation can be grouped into a commutator, though part of them (the imaginary ones) were initially parts of anticommutators. The remaining terms we presented as a collision integral of quasiparticles $\mathcal{L}$. This was done in order to have a much tighter connection with the microscopic theory of transverse dynamics in Fermi liquids. ${ }^{11}$

Note that the form of the interaction function (18) and (20), including its imaginary (pole) part, is not just some arbitrary equation used to reconfigure the "exact" transport equation from the Appendix. We will get exactly the same equation if we follow the microscopic description of transverse dynamics in (low-density) Fermi liquids at $T=0$ (Ref. 11) when the interaction function in the second order is equal to the irreducible vertex function (this vertex function is calculated in the second part of Refs. 11). Since the analytic definition of the interaction function via the exact irreducible vertex is more natural than simply as $P \frac{1}{\epsilon}$ terms in the collision operator, the imaginary pole terms should be included into the interaction function. What is more intriguing is that this interaction function (18) and (20), together with the quasiparticle collision integral (19) and (21), give an adequate description of transverse dynamics in the whole temperature range.

Such a self-consistent picture holds only up to the second order in the interaction. Beyond that the situa- 
tion is different, and the Fermi-liquid and classical kinetic pictures diverge completely. Unfortunately, there is no consistent diagrammatic derivation of the transverse transport equation in the third order for all temperatures similar to the second-order calculations in the Appendix. What is known concerns either the microscopic Fermi-liquid calculations at $T=0$, or the classic Boltzmann equation. However, in both cases the breakdown is caused by the retardation in the interaction.

At low temperatures, in the Fermi-liquid domain, the retardation manifests itself in the temporal nonlocality of the interaction. As is mentioned in the Introduction, the microscopic equation in the Green's function $G_{\uparrow \downarrow}(\omega, \mathbf{p} ; t, \mathbf{r})$ ( $t$ and $\mathbf{r}$ are "slow" hydrodynamic variables) can be reduced to the semiclassical transport equation in transverse distribution $n_{\uparrow \downarrow}(\mathbf{p} ; t, \mathbf{r})$ only if the shape of the $\delta$-type temporal peak in $G_{\uparrow \downarrow}(\omega, \mathbf{p})$ is preserved in dynamics. Only then the transition from a four-variable description by $G_{\uparrow \downarrow}(\omega, \mathbf{p})$ to the threevariable transport equation in $n_{\uparrow \downarrow}(\mathbf{p})$ is possible. Unfortunately, this is not the case starting from the third order in the interaction. ${ }^{11}$ In this case the $\delta$ peak splits into two peaks with different dynamics (roughly speaking, these peaks correspond to precession of tilted spin-ups and spin-downs). The $\delta$ shape of both peaks is preserved in dynamics, but, because of strong spin-up-spin-down asymmetry, the corresponding two coupled equations of motion are different and involve different combinations of peaks. In the case of homogeneous precession, these two peaks can be brought together by going to the rotating reference frame, when one recovers the standard equations. This procedure does not work in the case of spatially inhomogeneous precession when the frequency that could bring the peaks together depends on the coordinates and momenta. As a result, the closest thing to a transverse phenomenological transport equation which one can get is a set of two coupled equations in some partial transverse densities which reflect the presence of two distinct peaks in the Green's function $G_{\uparrow \downarrow}(\omega, \mathbf{p} ; t, \mathbf{r}$ ) (for details see Refs. 11). We want to emphasize that these problems arise only for transverse spin dynamics.

The problems in the high-temperature classical Boltzmann domain are different, though they also stem from the temporal nonlocality of the interaction. The attempts to go beyond the Boltzmann equation also demonstrated the importance of retardation and dissipative off-shell terms at the collisions. ${ }^{41}$ All the results obtained so far indicate that it is still possible to get a single closed kinetic equation in the distribution function, though these results were obtained for longitudinal dynamics where this fact is hardly surprising.

\section{ACKNOWLEDGMENTS}

One of the authors (A.M.) wants to thank the colleagues from the Laboratoire Kastler-Brossel de l'ENS for the hospitality during his stays in Paris. The work was supported by NSF Grant Nos. INT-9015836 and DMR-9412769 and an international cooperation grant from CNRS.

\section{APPENDIX}

Below we briefly describe the derivation of the kinetic equation for the transverse component of the distribution function using the Keldysh diagrammatic approach ${ }^{25}$ via nonequilibrium Green's functions $G_{\alpha \beta}^{+-}(\mathbf{p}, \mathbf{r}, t)$. The kinetic equation for a single-particle nonequilibrium Green's function $G_{12}^{-+} \equiv G_{\alpha_{1} \alpha_{2}}^{-+}\left(X_{1}, X_{2}\right)$ has the following form:

$$
\begin{aligned}
\left(\widehat{G}_{02}^{-1 *}-\widehat{G}_{01}^{-1}\right) G_{12}^{-+}= & -\operatorname{Tr}_{3}\left(\Sigma_{13}^{--} G_{32}^{-+}+\Sigma_{13}^{-+} G_{32}^{++}\right. \\
& \left.+G_{13}^{-+} \Sigma_{32}^{++}+G_{13}^{-} \Sigma_{32}^{-+}\right)
\end{aligned}
$$

where the coordinates $X_{i}$ are the time-spatial fourcoordinates $\left(\mathbf{r}_{i}, t_{i}\right), \alpha_{i}$ are the spin states, the trace assumes integration over the four-coordinates and summation over the spin states, $\Sigma_{i k} \equiv \Sigma_{\alpha_{i} \alpha_{k}}\left(X_{i}, X_{k}\right)$ are the single-particle self-energy functions, and the upper indices $\{+,-\}$ denote standard rules ${ }^{25}$ of ordering of $\Psi$ operators in the expressions for the corresponding Green's functions. The lhs of Eq. (A1) should reproduce the usual dynamic part of the transport equation, while the rhs gives the collision operator including molecular field and dissipative terms.

The time-spatial structure of the operator in the lhs of Eq. (A1) has the following form:

$$
\widehat{G}_{02}^{-1 *}-\widehat{G}_{01}^{-1}=-i\left(\frac{\partial}{\partial t_{1}}+\frac{\partial}{\partial t_{2}}\right)-\frac{1}{2 m}\left(\nabla_{1}^{2}-\nabla_{2}^{2}\right),
$$

where $m$ is the single-particle mass. The distribution function $n_{\alpha \beta}(\mathbf{p}, \mathbf{r}, t)[\alpha, \beta$ are the spin indices, $P=(\omega, \mathbf{p})$ is the four-momentum] can be expressed in terms of the Green's function $G_{\alpha \beta}^{-+}(X, \mathbf{P})$ as follows:

$$
n_{\alpha \beta}(\mathbf{p}, \mathbf{r}, t)=-i \int G_{\alpha \beta}(X, P) \frac{d \omega}{2 \pi} .
$$

After the Fourier transform, the lhs of Eq. (A1) reduces, with the help of Eqs. (A2) and (A3) at $t_{1}=t_{2}$, to a standard dynamic lhs of the Boltzmann kinetic equation, while the rhs contains all the relevant information on particle interaction and should reproduce the collision operator. This operator includes both dissipative and

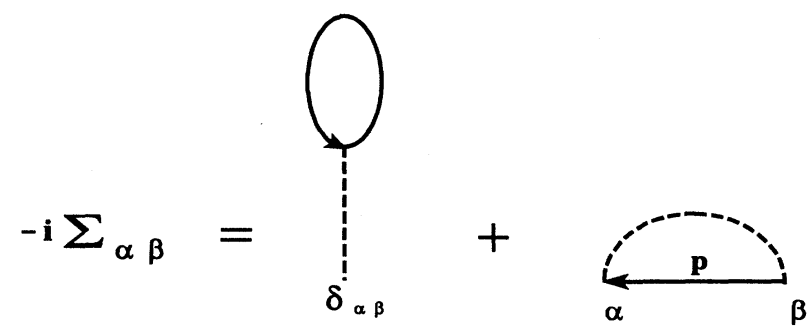

FIG. 1. Diagrams for the self-energy function $\Sigma$ in the first order in the interaction. 


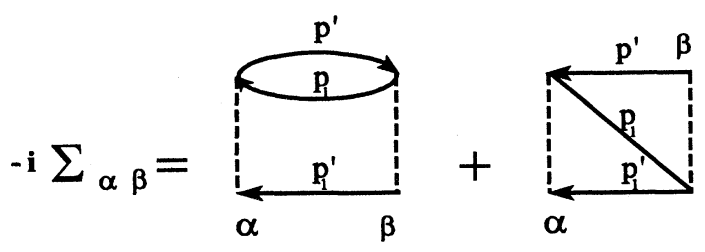

FIG. 2. Skeleton diagrams for the self-energy function $\Sigma$ in the second order in the interaction.

nondissipative terms, or, in other words, the dissipative collision integral and molecular field.

The derivation of the collision operator in the transport equation (A1) reduces to the calculation of self-energies $\Sigma$ and Green's functions $G$ by means of perturbation theory via the two-particle scattering $T$ matrix. If the interaction does not depend on spins and has an exchange origin,

$$
\begin{array}{r}
T_{\alpha \beta \mu \nu}\left(\mathbf{p}, \mathbf{p}^{\prime} ; \mathbf{p}_{1}, \mathbf{p}_{1}^{\prime}\right)=T\left(\mathbf{g}, \mathbf{g}^{\prime}\right) \delta_{\alpha \beta} \delta_{\mu \nu} \\
\mathbf{g}=\mathbf{p}_{1}-\mathbf{p}, \quad \mathbf{g}^{\prime}=\mathbf{p}+\mathbf{p}_{1}-2 \mathbf{p}^{\prime}
\end{array}
$$

As it was explained in Sec. II, we will restrict ourselves to calculations in the first and second orders in the interaction, i.e., scattering amplitude $\widehat{T}$.

Now let us separate the general collision operator into a molecular field and collision integral. The mean field, i.e., nondissipative coherent terms, correspond to a commutator of mass operators $\Sigma$ and Green's functions $G$ in the rhs of Eq. (A1) for $G_{\uparrow \downarrow}^{-+}$[arrows denote spin-up and spin-down states, and time-space coordinates are the same as in Eq. (A1)]:

$$
\begin{aligned}
L_{\text {coh }}=-\operatorname{Tr}_{3} l & {\left[G_{\uparrow \downarrow}^{-+} \operatorname{Re}\left(\Sigma_{\uparrow \uparrow}^{--}-\Sigma_{\downarrow \downarrow}^{--}\right)\right.} \\
& \left.+\operatorname{Re} \Sigma_{\uparrow \downarrow}^{--}\left(G_{\uparrow \uparrow}^{-+}-G_{\downarrow \downarrow}^{-+}\right)\right] .
\end{aligned}
$$

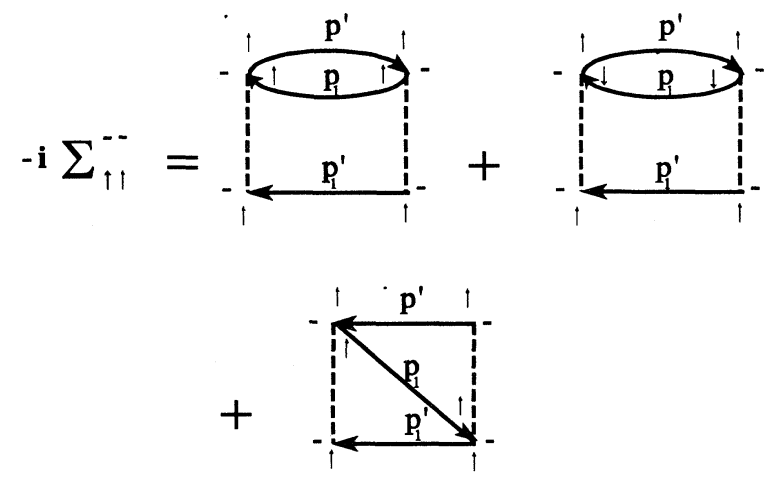

FIG. 3. The full set of diagrams for $\Sigma_{\uparrow \uparrow}^{--}$in the second order in the interaction.

Dissipative term or collision integral corresponds to the anticommutator of $\Sigma$ and $G$, and can be written as

$$
\begin{aligned}
L_{\text {coll }}= & \frac{1}{2} \operatorname{Tr}_{3}\left[\Sigma_{\uparrow \downarrow}^{+-}\left(G_{\uparrow \uparrow}^{-+}+G_{\downarrow \downarrow}^{-+}\right)+G_{\uparrow \downarrow}^{-+}\left(\Sigma_{\uparrow \uparrow}^{+-}+\Sigma_{\downarrow \downarrow}^{+-}\right)\right] \\
& -\frac{1}{2} \operatorname{Tr}_{3}\left[G_{\uparrow \downarrow}^{+-}\left(\Sigma_{\uparrow \uparrow}^{-+}+\Sigma_{\downarrow \downarrow}^{-+}\right)\right. \\
& \left.+\Sigma_{\uparrow \downarrow}^{-+}\left(G_{\uparrow \uparrow}^{+-}+G_{\downarrow \downarrow}^{+-}\right)\right] .
\end{aligned}
$$

In equilibrium, all spin matrices are diagonal. It is obvious that in the first order in the interaction, the collision integral (A6) is identically zero, and the only contribution to the rhs of the kinetic equation is given by the nondissipative mean-field term (A5).

The diagrams for $\Sigma$ in the first order in the interaction are given in Fig. 1. Direct calculation with two-particle interaction potential $U(\mathbf{p})$ yields

$$
-i \Sigma_{\uparrow \uparrow}^{--}=-i U(0) n+i \int U\left(\mathbf{p}-\mathbf{p}_{1}\right)\left[P \frac{1}{\omega_{1}-\epsilon_{1}+\mu}+i \pi\left[2 n_{\uparrow \uparrow}\left(\mathbf{p}_{1}\right)-1\right] \delta\left(\omega_{1}-\epsilon_{1}+\mu\right)\right] d^{4} p_{1}
$$

and similarly for $\Sigma_{\downarrow \downarrow}$ :

$$
\Sigma_{\uparrow \uparrow}^{--}-\Sigma_{\downarrow \downarrow}^{--}=-\frac{1}{\hbar} \int U\left(\mathbf{p}-\mathbf{p}_{1}\right) m\left(\mathbf{p}_{1}\right) \frac{d^{3} p_{1}}{(2 \pi \hbar)^{3}}
$$

[here and below $n(\mathbf{p}) \equiv n_{\uparrow \uparrow}^{(0)}(\mathbf{p})+n_{\downarrow \downarrow}^{(0)}(\mathbf{p})$ and $m(\mathbf{p}) \equiv n_{\uparrow \uparrow}^{(0)}(\mathbf{p})-n_{\downarrow \downarrow}^{(0)}(\mathbf{p})$ are the equilibrium density distributions for particles and the $z$ component of magnetic moment]. The calculations for $\Sigma_{\uparrow \downarrow}^{++}$are similar.

As was mentioned in Sec. II, all final results should be expressed via scattering amplitude $T$, and not via the potential $U$. This can be done with the help of the Lippmann-Schwinger equation (2). In the center of mass reference frame, the Lippmann-Schwinger equation has the form

$$
T(\mathbf{q}, \mathbf{k})=-U(\mathbf{k}-\mathbf{q})-M \int \frac{U\left(\mathbf{q}^{\prime}-\mathbf{q}\right) T\left(\mathbf{q}^{\prime}, \mathbf{k}\right)}{q^{\prime 2}-k^{2}-i 0} \frac{d^{3} q^{\prime}}{(2 \pi \hbar)^{3}}
$$


where $M=m / 2$ is the reduced mass, $\mathbf{q}$ and $\mathbf{k}$ are the relative momenta before and after the collision, $\mathbf{q}=\left(\mathbf{p}_{1}-\mathbf{p}\right) / 2$, $\mathbf{k}=\left(\mathbf{p}_{1}^{\prime}-\mathbf{p}^{\prime}\right) / 2$, and the scattering amplitude $T(\mathbf{q}, \mathbf{k})$ depends only on the momentum transfer, $\mathbf{k}-\mathbf{q}$. Since the collisions conserve the total momentum, $\mathbf{p}+\mathbf{p}_{1}=\mathbf{p}^{\prime}+\mathbf{p}_{1}^{\prime}$, Eq. (A9) can be rewritten as

$$
T\left(\mathbf{p}-\mathbf{p}^{\prime}\right)=-U\left(\mathbf{p}-\mathbf{p}^{\prime}\right)-M \int \frac{U\left(\mathbf{q}^{\prime}-\mathbf{p}_{1} / 2+\mathbf{p} / 2\right) T\left(\mathbf{p} / 2+\mathbf{p}_{1} / 2-\mathbf{p}^{\prime}-\mathbf{q}^{\prime}\right)}{q^{\prime 2}-k^{2}-i 0} \frac{d^{3} q^{\prime}}{(2 \pi \hbar)^{3}} .
$$

The solution of Eq. (A9) in the first and second orders in the interaction has the following form:

$$
U(\mathbf{k}-\mathbf{q})=-T(\mathbf{k}-\mathbf{q})+M \int \frac{T\left(\mathbf{q}^{\prime}-\mathbf{q}\right) T\left(\mathbf{k}-\mathbf{q}^{\prime}\right)}{q^{\prime 2}-k^{2}-i 0} \frac{d^{3} q^{\prime}}{(2 \pi \hbar)^{3}} .
$$

For quantum gases of long-wave particles (see Sec. III) it is sufficient to express both the $T$ matrix and the interaction via the $s$-wave scattering length $a$ [cf. Eq. (6)]:

$$
\begin{aligned}
& T(\mathbf{p})=-\frac{4 \pi}{M} \hbar^{2} a \\
& U(\mathbf{p})=\frac{4 \pi}{M} \hbar^{2} a\left[1-4 \pi \hbar^{2} P \int \frac{d^{3} q^{\prime}}{(2 \pi \hbar)^{3}} \frac{1}{q^{2}-k^{2}-i 0}\right] .
\end{aligned}
$$

As a result, the molecular field (A5) in the first order,

$$
L_{\mathrm{coh}}=\frac{2 \pi i}{\hbar} \int \frac{d^{3} p_{1}}{(2 \pi \hbar)^{3}} U\left(\mathbf{p}-\mathbf{p}_{1}\right)\left[n_{\uparrow \downarrow}\left(\mathbf{p}_{1}\right) m(\mathbf{p})-n_{\uparrow \downarrow}(\mathbf{p}) m\left(\mathbf{p}_{1}\right)\right]
$$

obtains the following form:

$$
L_{\mathrm{coh}}=-\frac{2 \pi i}{\hbar} \int \frac{d^{3} p_{1}}{(2 \pi \hbar)^{3}} T\left(\mathbf{p}-\mathbf{p}_{1}\right)\left[n_{\uparrow \downarrow}\left(\mathbf{p}_{1}\right) m(\mathbf{p})-n_{\uparrow \downarrow}(\mathbf{p}) m\left(\mathbf{p}_{1}\right)\right]
$$

The second-order terms include the second-order diagrams for $\Sigma$ as well as the second-order renormalization correction (A10) to the first-order diagrams. The skeleton diagrams for $\Sigma$ in the second order in the interaction are given in Fig. 2. The full set of the second-order diagrams for $\Sigma_{\uparrow \uparrow}^{--}$is given in Fig. 3; the diagrams for remaining components of $\Sigma$ are similar. The direct calculation of all corresponding integrals gives the following expression for $\Sigma_{\uparrow \uparrow}^{--}-\Sigma_{\downarrow \downarrow}^{--}$:

$$
\begin{aligned}
\Sigma_{\uparrow \uparrow}^{--}-\Sigma_{\downarrow \downarrow}^{--}= & \frac{2 M}{\hbar} \int \frac{d^{3} p^{\prime}}{(2 \pi \hbar)^{3}} \frac{d^{3} p_{1}}{(2 \pi \hbar)^{3}} P \frac{1}{2 m(\hbar \omega+\mu)+p_{1}^{2}-p^{\prime 2}-\mathbf{p}_{1}{ }^{2}}\left\{U^{2}\left(\mathbf{p}_{1}-\mathbf{p}^{\prime}\right) m\left(\mathbf{p}_{1}^{\prime}\right)\left[n\left(\mathbf{p}^{\prime}\right)-n\left(\mathbf{p}_{1}\right)\right]\right. \\
& -U\left(\mathbf{p}_{1}-\mathbf{p}^{\prime}\right) U\left(\mathbf{p}^{\prime}-\mathbf{p}\right) m\left(\mathbf{p}_{1}\right)+U\left(\mathbf{p}_{1}-\mathbf{p}^{\prime}\right) U\left(\mathbf{p}^{\prime}-\mathbf{p}\right)\left(m\left(\mathbf{p}_{1}\right) n\left(\mathbf{p}^{\prime}\right)\right. \\
& \left.\left.+m\left(\mathbf{p}^{\prime}\right)\left[n\left(\mathbf{p}_{1}\right)-n\left(\mathbf{p}_{1}^{\prime}\right)\right]\right)\right\}
\end{aligned}
$$

with $\mathbf{p}_{1}^{\prime}=\mathbf{p}+\mathbf{p}_{1}-\mathbf{p}^{\prime}$

As one can see from Eq. (A13), the first term in braces has a lower (second) order in density than all the rest, third-order, terms. However, this term is canceled out when we take into account the second-order renormalization corrections (A10) to the first order term in $\Sigma$. The renormalization correction (A10) to the mass operator (A8) has the form

$$
-\frac{M}{\hbar} P \int \frac{d^{3} \mathbf{k}}{(2 \pi \hbar)^{3}} \frac{d^{3} q}{(2 \pi \hbar)^{3}} \frac{T(\mathbf{k}+\mathbf{q}) T(\mathbf{k}-\mathbf{q})}{q^{2}-k^{2}} m(\mathbf{p}-2 \mathbf{k}) .
$$

This is exactly the same as the first term in braces (A13) if taken on the mass surface $\hbar \omega=\mu+p^{2} / 2 M$.

This cancellation is responsible for the disappearance of the so-called anomalous $I_{2}$ terms (terms with $\left.P \frac{1}{\epsilon}\right)$ from the molecular field in the Boltzmann-like equation when one is interested only in the second-order density terms in the collision operator (see discussion in Sec. III). Note that the $P \frac{1}{\epsilon}$ terms in the third order in density do not disappear from the molecular field (A14) even after renormalization of the interaction though these terms are obviously beyond the accuracy of the Boltzmann kinetic approach to high-temperature classical gases.

The final equation for the mean-field terms in the collision operator is 


$$
\begin{aligned}
L_{\mathrm{coh}}= & \frac{i \pi m}{\hbar} \int \frac{d^{3} p^{\prime}}{(2 \pi \hbar)^{3}} \frac{d^{3} p_{1}}{(2 \pi \hbar)^{3}} P \frac{1}{p^{2}+p_{1}^{2}-p^{2}-\left(\mathbf{p}+\mathbf{p}_{1}-\mathbf{p}^{\prime}\right)^{2}} \\
& \times\left\{T\left(\mathbf{p}_{1}-\mathbf{p}^{\prime}\right) T\left(\mathbf{p}^{\prime}-\mathbf{p}\right)\left\{n^{\prime}\left[n_{\uparrow \downarrow}\left(\mathbf{p}_{1}\right) m-m_{1} n_{\uparrow \downarrow}(\mathbf{p})\right]\right\}\right. \\
& \left.+\left[T^{2}\left(\mathbf{p}_{1}-\mathbf{p}^{\prime}\right)-T\left(\mathbf{p}_{1}-\mathbf{p}_{1}^{\prime}\right) T\left(\mathbf{p}^{\prime}-\mathbf{p}_{1}\right)\right]\left(n^{\prime}-n_{1}\right)\left[n_{\uparrow \downarrow}\left(\mathbf{p}_{1}^{\prime}\right) m-n_{\uparrow \downarrow}(\mathbf{p}) m\left(\mathbf{p}_{1}^{\prime}\right)\right]\right\}
\end{aligned}
$$

$\left[m=2 M\right.$ is the single-particle mass, $\left.n_{i} \equiv n\left(\mathbf{p}_{i}\right), m_{i} \equiv m\left(\mathbf{p}_{i}\right)\right]$, or, via the scattering length $a$,

$$
L_{\mathrm{coh}}=\frac{64 i a^{2} \pi^{3} \hbar^{3}}{m} \int \frac{d^{3} p^{\prime}}{(2 \pi \hbar)^{3}} \frac{d^{3} p_{1}}{(2 \pi \hbar)^{3}} P \frac{1}{p^{2}+p_{1}^{2}-p^{2}-\left(\mathbf{p}+\mathbf{p}_{1}-\mathbf{p}^{\prime}\right)^{2}} n^{\prime}\left[n_{\uparrow \downarrow}\left(\mathbf{p}_{1}\right) m-m_{1} n_{\uparrow \downarrow}(\mathbf{p})\right] .
$$

The analogous calculations for $\Sigma_{\uparrow \downarrow}^{-+}$and other diagrams in collision integral Eq. (A5) yield:

$$
\begin{aligned}
L_{\mathrm{coll}}= & \frac{\pi}{2 \hbar} \int \frac{d^{3} p_{1}}{(2 \pi \hbar)^{3}} \frac{d^{3} p^{\prime}}{(2 \pi \hbar)^{3}} \delta\left(\epsilon+\epsilon_{1}-\epsilon^{\prime}-\epsilon_{1}^{\prime}\right)\left\{T\left(\mathbf{p}_{1}-\mathbf{p}^{\prime}\right) T\left(\mathbf{p}_{1}^{\prime}-\mathbf{p}_{1}\right)\right\} \\
& \times\left\{\left(1-n^{\prime}\right)\left[n_{\uparrow \downarrow}\left(\mathbf{p}_{1}\right) n-n_{\uparrow \downarrow}(\mathbf{p}) n_{1}\right]+\frac{1}{2}\left[n_{\uparrow \downarrow}\left(\mathbf{p}_{1}\right)-n_{\uparrow \downarrow}(\mathbf{p})\right]\left(n^{\prime} n_{1}^{\prime}-m^{\prime} m_{1}^{\prime}\right)\right. \\
& \left.+\left[T^{2}\left(\mathbf{p}_{1}-\mathbf{p}^{\prime}\right)-T\left(\mathbf{p}_{1}-\mathbf{p}^{\prime}\right) T\left(\mathbf{p}_{1}^{\prime}-\mathbf{p}_{1}\right)\right]\left[\begin{array}{c}
n_{\uparrow \downarrow}\left(\mathbf{p}_{1}^{\prime}\right)\left[n n_{1}+n^{\prime}(2-n)-n^{\prime} n_{1}-m^{\prime} m_{1}\right] \\
-n_{\uparrow \downarrow}(\mathbf{p})\left[n^{\prime} n_{1}^{\prime}+n_{1}\left(2-n_{1}^{\prime}\right)-n^{\prime} n_{1}-m^{\prime} m_{1}\right]
\end{array}\right]\right\} .
\end{aligned}
$$

In the $s$-wave scattering approximation this equation simplifies to the form

$$
\begin{aligned}
L_{\mathrm{coll}}= & \frac{32 \pi^{3} \hbar^{3} a^{2}}{m^{2}} \int \frac{d^{3} p_{1}}{(2 \pi \hbar)^{3}} \frac{d^{3} p^{\prime}}{(2 \pi \hbar)^{3}} \delta\left(\epsilon+\epsilon_{1}-\epsilon^{\prime}-\epsilon_{1}^{\prime}\right) \\
& \times\left\{\left(1-n^{\prime}\right)\left[n_{\uparrow \downarrow}\left(\mathbf{p}_{1}\right) n-n_{\uparrow \downarrow}(\mathbf{p}) n_{1}\right]+\frac{1}{2}\left[n_{\uparrow \downarrow}\left(\mathbf{p}_{1}\right)-n_{\uparrow \downarrow}(\mathbf{p})\right]\left(n^{\prime} n_{1}^{\prime}-m^{\prime} m_{1}^{\prime}\right)\right\} .
\end{aligned}
$$

${ }^{1}$ E.P. Bashkin and A.E. Meyerovich, Adv. Phys. 30, 1 (1981).

${ }^{2}$ E.P. Bashkin, Sov. Phys. JETP Lett. 33, 8 (1981).

${ }^{3}$ C. Lhuillier and F. Laloe, J. Phys. (Paris) 43, 197; 43, 225 (1982).

${ }^{4}$ L.P. Levy and A. Ruckenstein, Phys. Rev. Lett. 52, 1519 (1984).

${ }^{5}$ A.E. Meyerovich, Phys. Lett. 107A, 177 (1985).

${ }^{6}$ E.P. Bashkin, Sov. Phys. JETP 66, 482 (1987).

7 J.W. Jeon and W.J. Mullin, J. Phys. (Paris) 49, 1691 (1988).

${ }^{8}$ J.W. Jeon and W.J. Mullin, Phys. Rev. Lett. 62, 2691 (1989).

9 A.E. Meyerovich, Phys. Rev. B 39, 9318 (1989).

${ }^{10}$ J.W. Jeon and W.J. Mullin, J. Low Temp. Phys. 88, 483 (1992).

${ }^{11}$ A.E. Meyerovich and K.A. Musaelian, J. Low Temp. Phys. 89, 781 (1992); 94, 249 (1994); 95, 789 (1994).

${ }^{12}$ A.E. Meyerovich, Helium Three, edited by W.P. Halperin and L.P. Pitaevski (Elsevier, Amsterdam, 1990), pp. 757879.

${ }^{13}$ L. Waldmann, Z. Naturforsch. 13a, 609 (1958); 15a, 19 (1960).

${ }^{14}$ R. Snider, J. Chem. Phys. 32, 1051 (1959).

${ }^{15}$ C.S. Wang Chang, G.E. Uhlenbeck, and J. de Boer, in Studies in Statistical Mechanics, edited by J. de Boer and G.E. Uhlenbeck (North-Holland, Amsterdam, 1964).

${ }^{16} \mathrm{~S}$. Hess, Z. Naturforsch. Teil A 22, 1871 (1967).

${ }^{17}$ A. Tip, Physica 52, 493 (1971); 53, 183 (1971).

${ }^{18}$ J.A. McLennan, Introduction to Non-Equilibrium Statistical Mechanics (Prentice-Hall, Englewood Cliffs, NJ, 1989).
${ }^{19}$ F. Laloe and W.J. Mullin, J. Stat. Phys. 59, 725 (1990).

${ }^{20}$ H. Moraal, Phys. Rep. 17, 225 (1975).

${ }^{21}$ V.P. Silin, Sov. Phys. JETP 6, 945 (1957).

${ }^{22}$ A.J. Leggett, J. Phys. C 3, 448 (1970).

${ }^{23}$ L.D. Landau, Sov. Phys. JETP 3, 920 (1956); 5, 101 (1957); 8, 70 (1958).

${ }^{24}$ A.A. Abrikosov, L.P. Gorkov, and I.E. Dzyaloshinski, Methods of Quantum Field Theory in Statistical Physics (Dover, New York, 1975).

${ }^{25}$ E.M. Lifshitz and L.P. Pitaevski, Physical Kinetics (Plenum, New York, 1979).

${ }^{26}$ G. Baym and C. Pethick, Landau Fermi Liquid Theory (Wiley, New York, 1991).

${ }^{27}$ R. Balian and C. de Domenicis, Ann. Phys. (N.Y.) 62, 229 (1971).

${ }^{28}$ J.M. Luttinger and Y.T. Liu, Ann. Phys. (N.Y.) 80, 1 (1973).

${ }^{29}$ C. Pethick and G.M. Carneiro, Phys. Rev. A 7, 304 (1973); Phys. Rev. B 11, 1106 (1975).

${ }^{30}$ M.B. Vetrovec and G.M. Carneiro, Phys. Rev. B 22, 1250 (1980).

${ }^{31} \mathrm{H}$. Keiter and G. Morandi, Phys. Rep. 109, 227 (1984).

${ }^{32}$ A.E. Ruckenstein and L.P. Levy, Phys. Rev. B 39, 183 (1989).

${ }^{33}$ A. Abrikosov and I.E. Dzyaloshinskii, Sov. Phys. JETP 8, 535 (1958).

${ }^{34}$ K.F. Quader and K.S. Bedell, J. Low Temp. Phys. 58, 89 (1985).

35 A.E. Meyerovich, Physica B 169, 183 (1991).

${ }^{36}$ A.E. Meyerovich and K.A. Musaelian, Phys. Rev. Lett. 72, 1710 (1994); Phys. Rev. B 47, 2897 (1993). 
${ }^{37}$ L.-J. Wei, N. Kalenchofsky, and D. Candela, Phys. Rev. Lett. 71, 879 (1993).

${ }^{38}$ J. Owers-Bradley, A. Child, and R.M. Bowley, Physica B 194-196, 903 (1994); J.H. Ager, R.M. Bowley, R. Konig, and J.R. Owers-Bradley, J. Low Temp. Phys. (to be published).

${ }^{39}$ E.P. Bashkin, Phys. Rev. Lett. 55, 1426 (1985).
${ }^{40}$ F. Laloe (private communication).

${ }^{41}$ F. Laloe, J. Phys. (Paris) 50, 1851 (1989); G. Tastevin, P.-J. Nacher, and F. Laloe, ibid. 50, 1879 (1989); P.-J. Nacher, G. Tastevin, and F. Laloe, ibid. 50, 1907 (1989).

${ }^{42}$ D.I. Golosov and A.E. Ruckenstein, Phys. Rev. Lett. 75 (1994). 